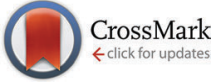

Cite this: Phys. Chem. Chem. Phys., 2017, 19, 2087

Received 13th September 2016, Accepted 4th December 2016

DOI: $10.1039 / c 6 c p 06312 h$

www.rsc.org/pccp

\title{
A density functional theory study of the carbon-coating effects on lithium iron borate battery electrodes $\dagger$
}

\begin{abstract}
Simon Loftager, Juan María García-Lastra and Tejs Vegge*
Lithium iron borate $\left(\mathrm{LiFeBO}_{3}\right)$ is a promising cathode material due to its high theoretical specific capacity, inexpensive components and small volume change during operation. Yet, challenges related to severe airand moisture-induced degradation have prompted the utilization of a protective coating on the electrode which also improves the electronic conductivity. However, not much is known about the preferential geometries of the coating as well as how these coating-electrode interfaces influence the lithium diffusion between the coating and the electrode. Here, we therefore present a density functional theory (DFT) study of the anchoring configurations of carbon coating on the $\mathrm{LiFeBO}_{3}$ electrode and its implications on the interfacial lithium diffusion. Due to large barriers associated with $\mathrm{Li}$-ion diffusion through a parallel-oriented pristine graphene coating on the $\mathrm{FeBO}_{3}$ and $\mathrm{LiFeBO}_{3}$ electrode surfaces, large structural defects in the graphene coating are required for fast Li-ion diffusion. However, such defects are expected to exist only in small concentrations due to their high formation energies. Alternative coating geometries were therefore investigated, and the configuration in which the coating layers were anchored normal to the electrode surface at $B$ and $O$ atoms was found to be most stable. Nudged elastic band (NEB) calculations of the lithium diffusion barriers across the interface between the optimally oriented coating layers and the electrode show no kinetic limitations for lithium extraction and insertion. Additionally, this graphite-coating configuration showed partial blocking of electrode-degrading species.
\end{abstract}

\section{Introduction}

Today's society is in urgent need of improved media for energy storage that can be used in portable electrics, electric vehicles and large-scale grid-based storage systems of renewable energy. In particular, batteries hold great potential as alternatives to limited and $\mathrm{CO}_{2}$-emitting fossil fuels for vehicular propulsion.

Despite being a high-capacity cathode material displaying excellent cyclability, $\mathrm{LiNi}_{1 / 3} \mathrm{Mn}_{1 / 3} \mathrm{Co}_{1 / 3} \mathrm{O}_{2}$ (NMC) is restricted by the toxicity and high cost of Co. Alternative cathode materials include the interesting $\mathrm{Li}_{2} \mathrm{VO}_{2} \mathrm{~F}$ compound showing excellent rate capability and high specific capacity, ${ }^{1}$ though currently limited by high price, and the transition-metal olivine-structured compound, $\mathrm{LiFePO}_{4}$, attracting interest due to its earth-abundant, environmentally benign elements, but is yet impeded by poor rate

Department of Energy Conversion and Storage, Technical University of Denmark, Fysikvej, 2800 Kgs. Lyngby, Denmark. E-mail: teve@dtu.dk

$\dagger$ Electronic supplementary information (ESI) available: Parallel graphene coating on $\mathrm{FeBO}_{3} / \mathrm{LiFeBO}_{3}$ electrodes; a stability analysis of $\mathrm{LiFeBO}_{3}$ surfaces; adsorption of $\mathrm{Li}$ ions on pristine graphene; NEB barriers for Li-ion diffusion through pristine and graphene defects; information of other anchoring configurations of the coating on the electrode surface; study of the diffusion of degradative species in graphite coating. See DOI: 10.1039/c6cp06312h capability stemming from a low ionic and electronic conductivity, ${ }^{2}$ which has so far only been improved by decreasing the $\mathrm{LiFePO}_{4}$ particle size, and decreasing the volumetric energy density.

In recent years, lithium iron borate $\left(\mathrm{LiFeBO}_{3}\right)$ has received increasing interest due to its low-cost constituents, a relatively high specific capacity of $220 \mathrm{~mA} \mathrm{~h} \mathrm{~g}{ }^{-1}$ and the very small volume change during operation of about $2 \%,{ }^{3}$ which lowers the risk of losing contact with the other battery components. However, pristine $\mathrm{LiFeBO}_{3}$ compounds are prone to oxidation from ambient air and moisture, and studies by Yamada et $a l^{3}$ and Bo et al. ${ }^{4,5}$ have clarified the detrimental effects of air oxidation on $\mathrm{LiFeBO}_{3}$ electrodes, which were shown to involve the displacement of $\mathrm{Fe}$ onto the $\mathrm{Li}$ sites as $\mathrm{Li}$ was leached out of the active $\mathrm{LiFeBO}_{3}$ material. ${ }^{4}$ Coating the $\mathrm{LiFeBO}_{3}$ electrode with a carbonaceous species has therefore become instrumental in protecting the electrode from oxidation and thereby improving the electrochemical performance of the material.

There exist numerous experimental studies which report on the beneficial properties endowed by carbon-coating the electrode material. Both hexagonal and monoclinic $\mathrm{LiMnBO}_{3}$ have been shown to benefit from being carbon-coated, showing a reduction in polarization and a correspondingly large increase in the reversible capacity from $65 \mathrm{~mA} \mathrm{~h} \mathrm{~g}^{-1}$ to $100 \mathrm{~mA} \mathrm{~h} \mathrm{~g}$ 
at $\mathrm{C} / 20 .{ }^{6} \mathrm{LiFePO}_{4}$ has also been shown to have its rate capability and reversible capacity greatly improved by carbon-coating the electrode, ${ }^{7-9}$ and a study by Zhou et al. ${ }^{10}$ in which $\mathrm{LiFePO}_{4}$ electrode particles were homogeneously and loosely wrapped with a graphene three-dimensional network suggested that this was attributed to smaller particle sizes, improved electronic conductivity due to higher graphitization and to an extended three-dimensional network graphene wrapping in which the voids between the graphene layers and the electrode easily enabled the diffusion of $\mathrm{Li}$ ions between the $\mathrm{LiFePO}_{4}$ crystal and the electrolyte.

In this computational study, the protective coating on the active $\mathrm{LiFeBO}_{3}$ electrode surface is modeled as carbon-coating layers on the $\mathrm{LiFeBO}_{3}$ electrode surface. The optimal anchoring was found to be that in which the coating-edge $\mathrm{C}$ atoms bond to electrode-surface $\mathrm{B}$ and $\mathrm{O}$ atoms and the coating layers are normal to the electrode surface. The analysis is combined with an assessment of the rate of lithium transport through different possible configurations of the carbon coatings, indicating no kinetic limitations to $\mathrm{Li}$ (de)insertion of the electrode across the optimal coating-electrode interface.

\section{Computational details}

\subsection{The crystal structure of $\mathrm{LiFeBO}_{3}$}

$\mathrm{LiFeBO}_{3}$ has a monoclinic crystal system in which $\mathrm{FeO}_{5}$ complexes link together at the edges forming chains along the $[-101]$ direction, and chains of $\mathrm{LiO}_{4}$ complexes run along the [001] direction. Boron and oxygen atoms form trigonal groups which connect the edge-sharing $\mathrm{FeO}_{5}$ chains. The crystal structure of $\mathrm{LiFeBO}_{3}$ is shown in Fig. 1. A commensurate modulation of $\mathrm{LiFeBO}_{3}$ has been experimentally observed by Janssen et al. ${ }^{11}$ which induces a minor displacement of the Li ions out of their trigonal bipyramidal planes resulting in an almost regular tetrahedral coordination of the $\mathrm{Li}$ ions to the neighboring $\mathrm{O}$ ions. This effectively doubles the unit cell of $\mathrm{LiFeBO}_{3}$ and alters the crystal symmetry from $C 2 / c$ to $C 2 / c(\alpha 0 \gamma) 00$ with $\alpha=1 / 2$ and $\gamma=0$. However, previous DFT studies ${ }^{12}$ have shown that the effect of

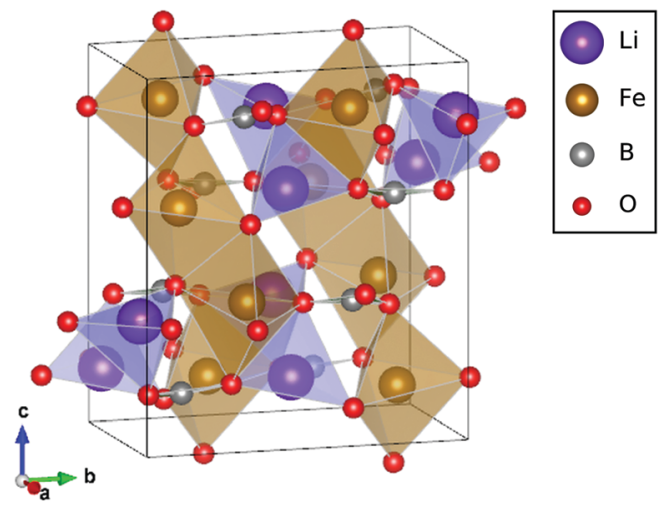

Fig. 1 Unit cell of $\mathrm{LiFeBO}_{3}$ with Fe atoms in the center of the brown edgesharing $\mathrm{FeO}_{5}$ complexes forming chains along the [-101] direction and $\mathrm{Li}$ atoms in purple $\mathrm{LiO}_{4}$ complexes forming chains in the [001] direction. Images were generated using the VESTA software package. ${ }^{13}$ this structural modulation has no influence on the activation barriers of $\mathrm{Li}$ ions and vacancies, and the modulation is therefore not included in the present study.

The $\mathrm{LiFeBO}_{3}$ structure was optimized starting from the experimental lattice constants reported by Janssen et al., ${ }^{11} a=$ $5.1350 \AA, b=9.0437 \AA, c=10.2907 \AA$ and $\beta=91.031^{\circ}$. As Li ions are extracted from $\mathrm{LiFeBO}_{3}$ during charge, the $\mathrm{Fe}^{2+}$ ions are oxidized to $\mathrm{Fe}^{3+}$. The $\mathrm{Fe}$ ions were consistently found to be in their high-spin state having their five $3 \mathrm{~d}$ orbitals occupied. Hence, Fe in $\mathrm{LiFeBO}_{3}$ is $\mathrm{Fe}^{2+}$ having a magnetic moment of $4 \mu_{\mathrm{B}}$ and $\mathrm{Fe}$ in $\mathrm{FeBO}_{3}$ is $\mathrm{Fe}^{3+}$ having a magnetic moment of $5 \mu_{\mathrm{B}}$.

\subsection{Computational methods}

In this study, input structures were built and analyzed using the Atomic Simulation Environment (ASE) package ${ }^{14}$ and structurally relaxed to their equilibrium structure by solving the electronicstructure problem within DFT. The DFT implementation in Vienna $\mathrm{Ab}$ initio Simulation Package (VASP) ${ }^{15}$ was used with plane waves being expanded up to a kinetic-energy cutoff of $500 \mathrm{eV}$. The projector-augmented-wave (PAW) method $^{16}$ was used to model the atomic cores, and in the PAW potentials, for $\mathrm{Li}, \mathrm{B}$ and $\mathrm{O}$ the $1 \mathrm{~s}$ electrons were treated as core electrons, and for Fe the 1s, 2s, 2p, 3s and $3 p$ electron shells were treated as core electrons. The exchange and correlation effects were modeled within the generalized-gradient approximation (GGA) by the Perdew-Burke-Ernzerhof (PBE) parametrization. ${ }^{17}$ The partial occupations were determined using the tetrahedron method with Blöchl corrections, and the electronic levels were smeared by $0.05 \mathrm{eV}$.

Due to the incomplete cancellation of the electron selfinteraction (SI) error inherent in the GGA, calculated properties, such as the band gap, can deviate significantly from experimental values, ${ }^{18,19}$ stemming from the SI-induced delocalization of the electrons within this model, especially for systems having strongly localized d-orbital electrons, such as $\mathrm{LiFeBO}_{3}$. Previous studies have efficiently rectified the SI error in the GGA by employing the Hubbard- $U$ correction scheme ${ }^{20}$ and following the work by Seo et al. ${ }^{21}$ a Hubbard $U$ of $4.3 \mathrm{eV}$ in the rotationally invariant scheme from the study of Dudarev et $a .^{22}$ was applied on the $3 \mathrm{~d}$ orbitals of $\mathrm{Fe}$ in $\mathrm{FeBO}_{3}$ and $\mathrm{LiFeBO}_{3}$. To correctly model the van der Waals (vdW) interaction between the graphene layers, a vdW-based functional should in principle be employed. Only a few previous studies $^{9,23}$ have combined Hubbard corrections with a vdW functional, however, it is not yet a well-established, widely used approach to employ such $\mathrm{vdW}+U$ functionals. Generally, the error introduced by having a slightly incorrect coating-layer separation is not expected to be significant. For instance, by stretching graphite along the layer stacking $(c)$ direction and changing the experimentally observed layer separation from $3.39 \AA$ to $4.94 \AA$ (corresponding to half of the $b$ lattice parameter for $\mathrm{FeBO}_{3}$ used later in this study as the coating-layer separation), the energy of the system per $\mathrm{C}$ atom only changed by $9.6 \mathrm{meV}$. Likewise, the interaction between the parallel graphene coating and the $\mathrm{FeBO}_{3} / \mathrm{LiFeBO}_{3}$ electrodes (see Fig. 2) is expected to be small, as the coating-electrode separation is large: a fit to the shifted total energies in Fig. S1(a) (ESI $\dagger$ ) calculated using the BEEF-vdW functional ${ }^{24}$ with the Hubbard- $U$ correction 
(a)

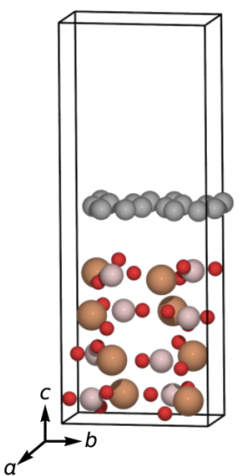

(b)

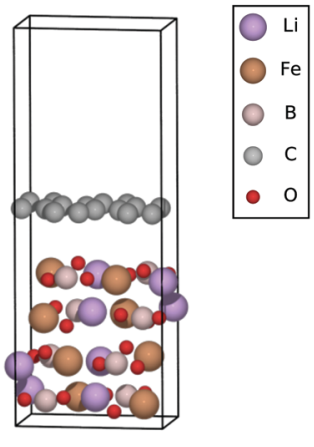

Fig. 2 Parallel-oriented coating geometry on the fully delithiated electrode (a) and the fully lithiated electrode (b).

(on the Fe:3d orbitals) shows that the optimal coating-FeBO $\mathrm{F}_{3} /$ $\mathrm{LiFeBO}_{3}$ separation is $3.7 \AA$. This is only slightly smaller than that predicted by the $\mathrm{PBE}+U$ functional when the coatingelectrode interactions become negligible at about $4.0 \AA$ as seen in Fig. S1(b) (ESI $\uparrow$ ). However, as previously stated the combination of a vdW functional and Hubbard corrections has not been thoroughly tested yet, and we have therefore adhered to the widely deployed $\mathrm{PBE}+U$ functional.

The unit cell and internal coordinates of $\mathrm{FeBO}_{3}$ and $\mathrm{LiFeBO}_{3}$ were fully relaxed to their equilibrium geometry such that the forces on the atoms did not exceed $0.05 \mathrm{eV} \AA^{-1}$ using the Fast Inertial Relaxation Engine (FIRE) minimization algorithm ${ }^{25}$ and the Brillouin zone was sampled using a Monkhorst-Pack (MP) mesh of $4 \times 2 \times 2$. The simulation cell used in the subsequent relaxation of the coating-electrode structures was inherited from the unit-cell dimensions of $\mathrm{FeBO}_{3}$ and $\mathrm{LiFeBO}_{3}$ for Li-ion and Li-vacancy calculations, respectively, with vacuum added in the $c$-direction so as to form a (001) surface of the $\mathrm{FeBO}_{3} / \mathrm{LiFeBO}_{3}$ electrodes and the two bottommost electrode layers were kept spatially fixed. For such supercells, an MP mesh of $4 \times 2 \times 1$ was used. In calculations simulating Li insertion or Li-vacancy formation, the aforementioned supercells were doubled in the $a$-direction and a $2 \times 2 \times 1 \mathrm{MP}$ mesh was used. The two bottom layers of the coated electrodes were fixed. For all structure relaxations, the self-consistent solution to the KohnSham equation was performed with an accuracy of $0.1 \mathrm{meV}$.

For the determination of the activation barriers of the Li-ion diffusion across the coating-electrode interface, the nudged elastic band (NEB) method ${ }^{26}$ implemented in ASE was utilized with a total number of seven images. The initial Li-ion pathway was created by linearly interpolating between the initial and final images. The energies were calculated using VASP, and the forces were let to relax to the same threshold value as for the structure relaxations $\left(0.05 \mathrm{eV}^{-1}\right)$ using the FIRE algorithm.

\section{Results and discussion}

\subsection{DFT-optimized lattice of bulk $\mathrm{LiFeBO}_{3}$ and $\mathrm{FeBO}_{3}$}

Upon relaxation of all internal degrees of freedom, the lattice parameters for the $\mathrm{LiFeBO}_{3}$ unit cell were found to be $a=5.168 \AA$, $b=9.109 \AA, c=10.349 \AA$ and $\beta=91.28^{\circ}$ in good agreement with experimental values ${ }^{11}$ (see above). The lattice parameters of the $\mathrm{FeBO}_{3}$ unit cell were found to be $a=5.308 \AA, b=8.989 \AA$, $c=$ $10.188 \AA$ and $\beta=89.40^{\circ}$ giving rise to a volume contraction upon fully delithiating $\mathrm{LiFeBO}_{3}$ to $\mathrm{FeBO}_{3}$ of $0.2 \%$.

\subsection{Parallel-oriented coating on $\mathrm{FeBO}_{3} / \mathrm{LiFeBO}_{3}$ electrodes}

Graphene sheets oriented parallel to metal surfaces have previously been found to bind via chemisorption or physisorption depending on the substrate ${ }^{9,27}$ and we have therefore investigated the effects of arranging the graphene sheet parallel to the surfaces of the $\mathrm{FeBO}_{3}$ and $\mathrm{LiFeBO}_{3}$ electrodes.

In order to determine the most stable low-index surface of the $\mathrm{LiFeBO}_{3}$ electrode, the surface energies, listed in Table 1, were calculated for the lowest-index surfaces, i.e. the (100), (010) and (001) $\mathrm{LiFeBO}_{3}$ surfaces. It is seen that the (001) surface is approximately three times more stable $\left(15 \mathrm{meV} \AA^{-2}\right)$ as the other two surfaces ( 41 and $47 \mathrm{meV} \AA^{-2}$ ). The surfaces are shown in Fig. S2 (ESI $\dagger$ ). It is therefore expected that the (001) surface will be the most dominant surface involved in the formation of external interfaces.

The surfaces of some oxide materials, ${ }^{28,29}$ such as $\mathrm{LiNi}_{x} \mathrm{Mn}_{x^{-}}$ $\mathrm{Co}_{1-x} \mathrm{O}_{2}$ and $\mathrm{Li}_{2} \mathrm{MnO}_{3}$, are known to display reconstruction. However, in this study no indications of such surface reconstruction were observed for the $\mathrm{FeBO}_{3}$ or $\mathrm{LiFeBO}_{3}$ electrodes. Yet, it should be noted that surface reconstruction often exists over large areas which could not be captured by our relatively small simulation cells.

The (001) surface of the $\mathrm{FeBO}_{3}$ electrode can be covered by a pure graphene sheet with an expansive strain on graphene of only $7.5 \%$ along the $a$-axis and $5.1 \%$ along the $b$-axis with a resulting strain energy of $239 \mathrm{meV}$ per $\mathrm{C}$ atom, and the (001) surface of the $\mathrm{LiFeBO}_{3}$ electrode can be covered by a pure graphene sheet with an expansive strain of only $4.7 \%$ along the $a$-axis and $6.5 \%$ along the $b$-axis with a resulting strain energy of $192 \mathrm{meV}$ per C. However, due to the large separation between the parallel-oriented graphene coating and the $\mathrm{FeBO}_{3} / \mathrm{LiFeBO}_{3}$ surfaces of $4.0 \AA$ ( $3.7 \AA$ if vdW effects are taken into account; see S1 in the ESI $\dagger$ ), as seen in Fig. 2, the interaction between the coating and the electrodes is found to be very small. Therefore, the two subsystems will be treated separately for the case of having the graphene coating parallel to the (001) electrode surface.

The surface lithiation/delithiation processes can therefore to a first approximation be analyzed by studying the surface electrodes shown in Fig. 3.

As listed in Table 2, for the bulk electrode the insertion energy is $3.26 \mathrm{eV}$ and the vacancy-formation energy is $2.90 \mathrm{eV}$ versus $\mathrm{Li}(\mathrm{s})$ with the bulk equilibrium voltage being $3.08 \mathrm{~V}$

Table 1 Surface energies for low-index surfaces of the $\mathrm{LiFeBO}_{3}$ electrode

\begin{tabular}{ll}
\hline Surface & Surface energy $\left[\mathrm{meV}^{-2}\right]$ \\
\hline$(100)$ & 41 \\
$(010)$ & 47 \\
$(001)$ & 15
\end{tabular}


(a)

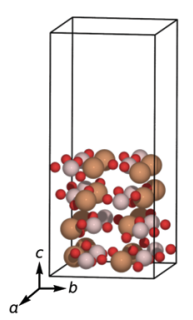

(c)

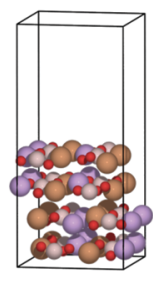

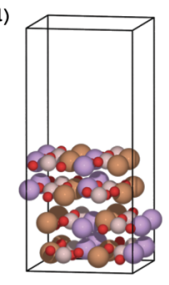

Fig. $3 \mathrm{Li}_{x} \mathrm{FeBO}_{3}$ electrodes with exposed (001) surfaces: (a) the fully delithiated (charged) phase. (b) The delithiated phase at initial stage of discharge, containing only one Li ion. (c) The lithiated phase at final stage of discharge, containing only one Li vacancy. (d) The fully lithiated (discharged) phase. The coloring of the atoms follows that of Fig. 2.

versus $\operatorname{Li}(\mathrm{s})$. The presence of the (001) surfaces shown in Fig. 3 makes it more favorable for the $\mathrm{Li}$ ions to be inserted in the outermost layer of the electrode as the insertion energy is $3.47 \mathrm{eV}$ and the vacancy-formation energy is $3.22 \mathrm{eV}$, i.e. higher than those for the bulk electrode. In a calculation, a $\mathrm{Li}$ ion initially $3 \AA$ above the $\mathrm{LiFeBO}_{3}$ electrode surface was seen to absorb into the surface layer of the electrode with no kinetic barriers to overcome. The energy gain $(3.47 \mathrm{eV})$ of this process is much larger than the $\mathrm{Li}$ adsorption energy on pristine graphene, $1.7 \mathrm{eV}$ as shown in Fig. S3 (ESI $\dagger$ ), suggesting that a $\mathrm{Li}$ ion adsorbed on the graphene coating on the electrode side would presumably have a negligible kinetic barrier toward insertion of the Li ion into the electrode surface layer. On the other hand, it is well known that the diffusion barrier of the Li ion through pristine graphene is very high, ${ }^{30}$ warranting further investigations into the effect of the graphene defects on the Li diffusion through the coating.

Li transport through the hollow site of pristine graphene, through graphene with a monovacancy and through graphene with a 5-8-5 divacancy was investigated using the NEB method which yielded the results shown in Fig. S4 (ESI $\dagger$ ). As listed in Table 3, the barrier for Li-ion diffusion through a hollow site of pristine graphene is $7.41 \mathrm{eV}$, agreeing well with a previous study. ${ }^{31}$ The barrier for Li diffusion through a graphene monovacancy is $2.95 \mathrm{eV}$, through a Stone-Wales defect $3.73 \mathrm{eV}$ and through a graphene 5-8-5 divacancy $1.11 \mathrm{eV}$. The electrochemical driving force induced by the presence of the $\mathrm{FeBO}_{3} / \mathrm{LiFeBO}_{3}$ electrodes, i.e. maximum $3.47 \mathrm{eV}$, depending on the specific location of the transition state, is therefore insufficient to lower the energy barrier for Li diffusion through a perfect graphene/ graphite layer to support the current densities under normal charge/discharge conditions. The same conclusions can be made for

Table 2 Formation energies and electrode potentials versus metallic lithium. The insertion energy is calculated as $E_{\mathrm{ins}}=\left[E_{\mathrm{Li}_{0.5} \mathrm{FeBO}_{3}}-\left(E_{\mathrm{FeBO}_{3}}+\right.\right.$ $\left.\left.8 E_{\mathrm{Li}(\mathrm{s})}\right)\right] /(-8)$, the vacancy-formation energy as $E_{\mathrm{VF}}=\left[E_{\mathrm{LiFeBO}_{3}}-\left(E_{\mathrm{Li}_{0} \mathrm{FeBO}_{3}}+\right.\right.$ $\left.\left.8 E_{\mathrm{Li}(\mathrm{s})}\right)\right] /(-8)$ and the equilibrium potential as $U_{\text {eq }}=\left[E_{\mathrm{LiFeBO}_{3}}-\left(E_{\mathrm{FeBO}_{3}}-\right.\right.$ $\left.\left.16 E_{\mathrm{Li}(\mathrm{s})}\right)\right] /(-16 e)$

\begin{tabular}{llll}
\hline & $\begin{array}{l}\text { Insertion } \\
\text { energy }[\mathrm{eV}]\end{array}$ & $\begin{array}{l}\text { Vacancy-formation } \\
\text { energy }[\mathrm{eV}]\end{array}$ & $\begin{array}{l}\text { Equilibrium } \\
\text { potential }[\mathrm{V}]\end{array}$ \\
\hline Bulk & 3.26 & 2.90 & 3.08 \\
Surface & 3.47 & 3.22 &
\end{tabular}

Table 3 Formation energies of the most plausible defects in graphene calculated as the difference between the pristine graphene and the defected graphene with respect to $C$ in graphene, i.e. $E_{\text {form }}=E_{\text {pristine }}-$ $\left(E_{\text {defected }}+N_{\text {defect-C }} E_{C \text { (graphene) }}\right)$, where $E_{\text {pristine }}$ is the total energy of the pristine graphene sheet, $E_{\text {defected }}$ is the total energy of the defected graphene sheet, $N_{\text {defect-C }}$ is the number of $C$ atoms removed in order to form the defect and $E_{C(\text { graphene) }}$ is the total energy of $C$ in graphene. These values are in good agreement with literature values. ${ }^{32-34}$ Also listed are the barriers for Li diffusion through the defects

\begin{tabular}{lll}
\hline Defect type & $\begin{array}{l}\text { Formation } \\
\text { energy }[\mathrm{eV}]\end{array}$ & $\begin{array}{l}\text { Activation barrier of } \\
\text { Li diffusion }[\mathrm{eV}]\end{array}$ \\
\hline Stone-Wales defect & 5.19 & 3.73 \\
Monovacancy & 7.84 & 2.95 \\
5-8-5 divacancy & 7.69 & 1.11
\end{tabular}

structural defects like monovacancies and Stone-Wales defects, and our findings show that only larger defects, e.g. 5-8-5 divacancies, can become active with a transport barrier of $1.11 \mathrm{eV}$. However, due to the high formation energy of these defects-see Table 3-their equilibrium concentration will be low under most conditions and alternative coating geometries and Li-ion pathways must therefore also be considered.

\subsection{Misaligned coating on $\mathrm{FeBO}_{3} / \mathrm{LiFeBO}_{3}$ electrodes}

Transmission electron microscopy (TEM) images on carbon-coated $\mathrm{LiFeBO}_{3}$ nanoparticle electrodes obtained by Zhang et al. ${ }^{35}$ show that a significant misalignment exists between the electrode particles and 1-3 monolayers of carbon coating (Fig. 4(d) in the study of Zhang et al.), and similar observations were reported by Tao et al. ${ }^{36}$ on carbon-coated $\mathrm{LiFeBO}_{3}$ nanoparticles (Fig. 9(b) in the study of Tao et al.). We have therefore investigated the effects of an angular misalignment of the carbon-coating layers on the $\mathrm{FeBO}_{3}$ and $\mathrm{LiFeBO}_{3}$ electrodes. The coating is here modeled as graphite layers whose edges opposite to the electrode are terminated by hydrogen atoms to avoid dangling bonds. Various positions on the electrode surface at which the coating could anchor were investigated using one graphite layer per unit cell, placed normal to the electrode surface. The graphene coating was strained expansively to the electrode unit cell and the strain of the graphite layer in the electrode $a$-direction was $7.5 \%$ for the $\mathrm{FeBO}_{3}$ electrode yielding a strain energy (i.e. the energy difference between unstrained and strained graphene coating) of $141 \mathrm{meV}$ per $\mathrm{C}$ and $4.7 \%$ for the $\mathrm{LiFeBO}_{3}$ electrode with a strain energy of $62 \mathrm{meV}$ per C. For both electrodes, the most stable anchoring configuration was found to be that in which one of the coatingedge $\mathrm{C}$ atoms bonded to a $\mathrm{B}$ atom with a bond length of $1.63 \AA$ and $1.60 \AA$ for $\mathrm{FeBO}_{3}$ and $\mathrm{LiFeBO}_{3}$, respectively, and the other coating-edge $\mathrm{C}$ atom bonded to an $\mathrm{O}$ atom with a bond length of $1.27 \AA$ and $1.36 \AA$ for $\mathrm{FeBO}_{3}$ and $\mathrm{LiFeBO}_{3}$, respectively, as shown in Fig. 4, indicating that the graphite coating is chemisorbed on the electrode surface. This anchoring arrangement was found to be $284 \mathrm{meV}$ and $470 \mathrm{meV}$ per surface-bonding $\mathrm{C}$ atom (four per unit cell) more stable than the second-lowest-energy anchoring configuration in which the $\mathrm{C}$ atom which previously bonded to the $\mathrm{B}$ atom now bonded to an $\mathrm{Fe}$ atom in $\mathrm{FeBO}_{3}$ and $\mathrm{LiFeBO}_{3}$, respectively, shown in Fig. S5 (ESI $\dagger$ ). 
(a)



(b)

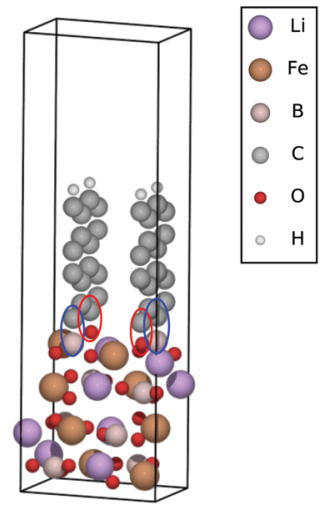

Fig. 4 Most stable anchoring configuration of the coating-electrode interface in which the coating-edge $\mathrm{C}$ atoms (two per coating layer) bond to a surface $\mathrm{B}$ atom, indicated by blue ellipses, and a surface $\mathrm{O}$ atom, indicated by red ellipses, in both $\mathrm{FeBO}_{3}$ (a) and $\mathrm{LiFeBO}_{3}$ (b).

The relative stability of the coating-electrode interface versus the angle between the carbon coating and the electrode is shown in Fig. 5. From here the optimal angular configuration between the electrode (001) surface and the coating plane hinged to the aforementioned $\mathrm{B}$ atom and the $\mathrm{O}$ atom was found to be that in which the graphene coating was positioned normal to the electrode surface (vide Fig. 4). The distance between the coating layers in the $b$-direction was $4.49 \AA$ for $\mathrm{FeBO}_{3}$ and $4.55 \AA$ for $\mathrm{LiFeBO}_{3}$. In this optimal configuration the graphite layers are stacked as AA along the $b$-axis. Calculations on a structure possessing only one coating layer per unit cell—resulting in a layer spacing of the $b$ lattice parameter-showed identical results, implying that having a less dense distribution

(a)

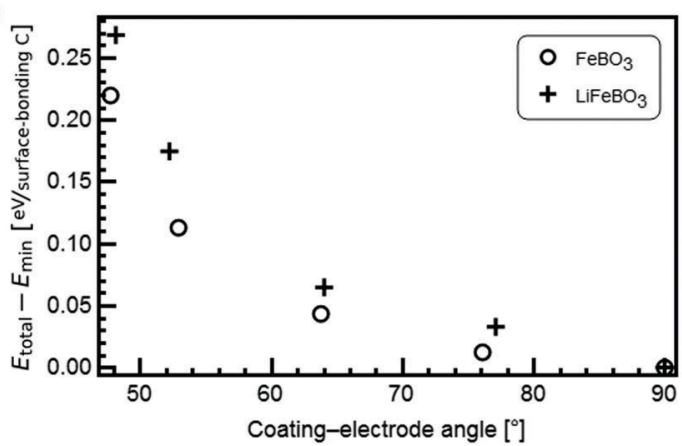

(b)
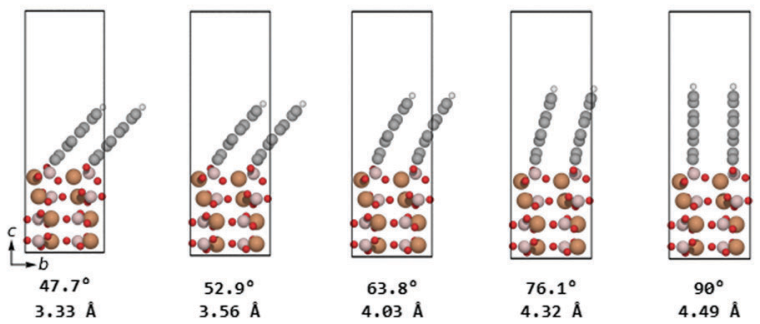

Fig. 5 (a) Stability of the carbon coating on the electrode as a function of the angle between the coating and the electrode surface as shown in (b) for $\mathrm{FeBO}_{3}$ with coating-electrode angles and coating interlayer distances listed below. The coloring of the atoms follows that of Fig. 4 . of coating layers on the $\mathrm{FeBO}_{3} / \mathrm{LiFeBO}_{3}$ surface does not alter the predictions of the angular coating orientation made by the aforementioned study with two graphite layers per unit cell.

Using the optimal coating-electrode geometry shown in Fig. 4, the potential of a $\mathrm{Li}$ ion in a graphitic anode, in the graphite coating attached to the electrode, in the coated electrode surface layer and in bulk $\mathrm{FeBO}_{3}$ during initial-stage discharge was calculated and is depicted in Fig. 6(a). A potential drop of $2.22 \mathrm{~V}$ during Li-ion diffusion from the hollow site of graphite closest to the electrode surface into the surface layer of the electrode was found. Interestingly, a $\mathrm{Li}$ ion stabilizes in the surface layer of $\mathrm{FeBO}_{3}$ compared to being inserted into bulk $\mathrm{FeBO}_{3}$ by $0.17 \mathrm{eV}$. However, as more $\mathrm{Li}$ ions are inserted from the graphite coating, the $\mathrm{Li}$ ion in the electrode surface layer is pushed further into the interior of the positive electrode due to the $\mathrm{Li}-\mathrm{Li}$ repulsion. Additionally, the electric-potential landscape for a Li ion during final-stage discharge is mapped out in Fig. 6(b). Here, it is noteworthy that the potential of the $\mathrm{Li}$ ion inserted into the electrode surface layer (versus metallic $\mathrm{Li}$ ) is $1.12 \mathrm{~V}$ smaller than the potential of a $\mathrm{Li}$ ion inserted into the vacancy-containing bulk electrode. This implies that a significant part of the experimentally observed sloping of the voltage profile near the end of discharge in $\mathrm{LiFeBO}_{3}$ could come from the difference between the surface and bulk potentials.

In order to determine possible interface-induced kinetic limitations for Li-ion transport between the graphite coating and the electrodes, NEB calculations were carried out at both initial-stage and final-stage discharge. The NEB results in Fig. 8

(a)

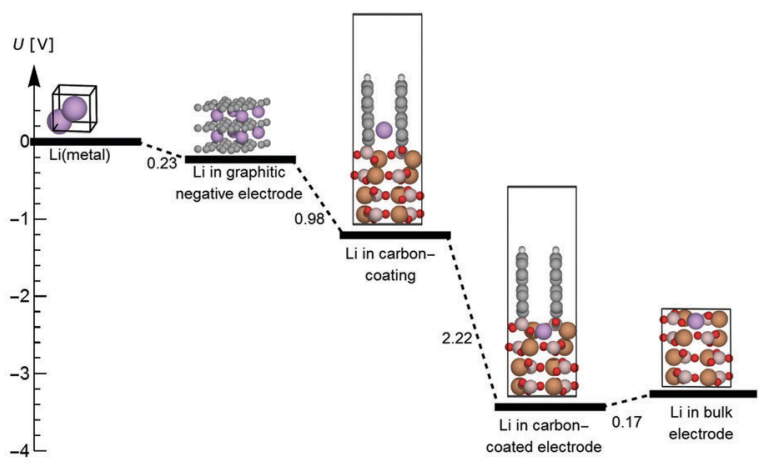

(b)

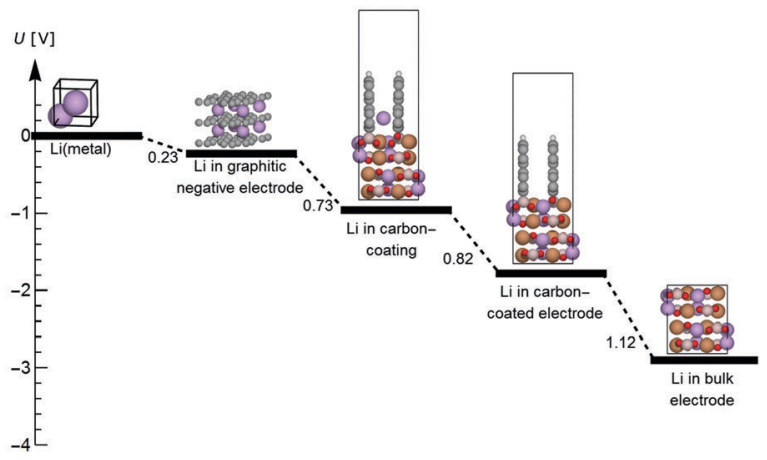

Fig. 6 Landscape of the electric potential of Li-ion insertion into the (a) $\mathrm{FeBO}_{3}$ positive-electrode material during the initial discharge process and (b) vacancy-containing $\mathrm{LiFeBO}_{3}$ positive-electrode material during the final discharge process. The coloring of the atoms follows that of Fig. 4. 
show that there are no activation barriers for Li-ion transport across the coating-electrode interface during (a) initial-stage and (b) final-stage discharge (a barrier of $27 \mathrm{meV}$ predicted to exist at the final-stage discharge is easily overcome by thermal excitation of the $\mathrm{Li}$ ions at room temperature). In both the charged and discharged configurations, local minima were identified for the Li ion in the graphene coating as shown in the insets in Fig. 7. Hence, the angular configuration of the graphite coating shown in Fig. 4 is found to provide an energetically preferred state with significantly reduced kinetic barriers for lithium to the positive electrode, compared to the parallel graphene coatings (vide Fig. 2).

In a realistic situation the size of the graphene sheets is sufficiently large to expect that the interlayer distance between graphene sheets when they are far from the $\mathrm{FeBO}_{3} / \mathrm{LiFBO}_{3}$ electrode will correspond to that of graphite (i.e. $3.39 \AA$ A). Looking at Fig. 5, in order to reach this situation we can hypothesize two scenarios: (i) the graphene sheets are anchored perpendicularly to the $\mathrm{FeBO}_{3} / \mathrm{LiFBO}_{3}$ electrode, having the graphene interlayer distance of $4.49 \AA$ at the anchoring position. Then the sheets gradually bend until they end up being a distance of $3.39 \AA$ apart. (ii) The graphene sheets are tilted $\sim 48^{\circ}$ at the anchoring position, having their interlayer

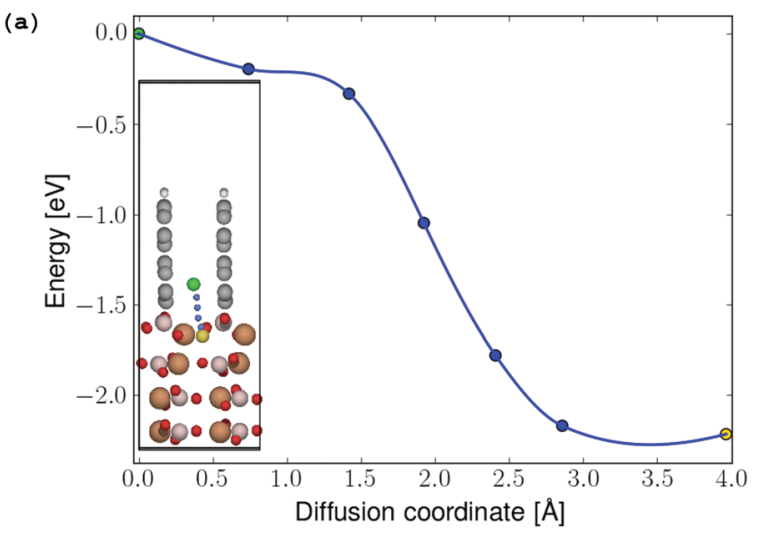

(b)



Fig. 7 (a) Energy profile for Li-ion diffusion from the graphene coating into the $\mathrm{FeBO}_{3}$ and (b) $\mathrm{LiFeBO}_{3}$ electrode, respectively, with the diffusion pathway shown in the insets. The diffusing Li ion is colored in green (initial position), blue (intermediate positions) and yellow (final position). The coloring of the rest of the atoms follows that of Fig. 4.

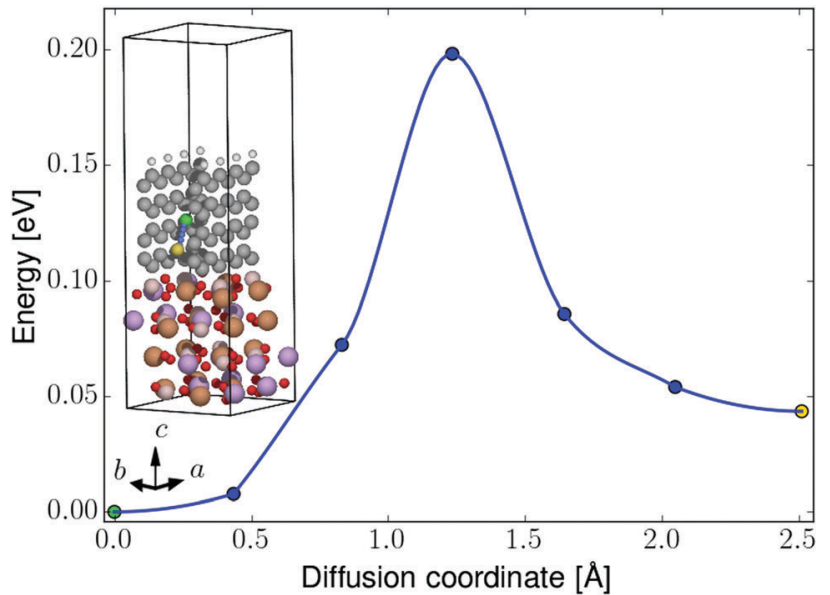

Fig. 8 NEB barrier for $\mathrm{Li}$-ion diffusion between graphene hollow sites in the perpendicular-oriented coating on the $\mathrm{LiFeBO}_{3}$ electrode. The diffusing Li ion is colored in green (initial position), blue (intermediate positions) and yellow (final position). The coloring of the rest of the atoms follows that of Fig. 4

distance $3.39 \AA$ from the starting point. It is difficult to assess which of the two situations will occur in reality since in the latter scenario it is necessary to provide $\sim 0.25 \mathrm{eV}$ per $\mathrm{C}$ atom to tilt the graphene sheets $48^{\circ}$ while in the former situation it is necessary to spend some energy to bend the graphene. Analyzing the energy associated with the bending of the graphene sheets is not a trivial task as it has been reported in a recent study by Nilsson et al. ${ }^{37}$ which combines DFT with experiments, showing that the bending of graphene sheets on top of metallic substrates is strongly dependent on which atoms saturate the edges of the graphene sheets. In any case, even in the scenario of an angling of the whole coating layers around the anchoring sites on the electrode surfaces (as in Fig. 5(b)), the facile Li diffusion across the coating-electrode interface in Fig. 7 is not expected to be impeded due to the relatively open Li pathway across the interface, which persists even for significant angling, such as $47.7^{\circ}$ in Fig. 5(b). In this tilted geometry, the Li-ion diffusion far away from the electrode would resemble that of diffusion in graphite, where a relatively low activation barrier for a $\mathrm{Li}$ ion jumping between hollow sites of $0.47 \mathrm{eV}$ was found, agreeing well with previous findings. ${ }^{38}$ Despite the successful application of carbon-coating the $\mathrm{LiFeBO}_{3}$ electrodes, these materials are still observed to undergo degradation to some extent. In general, the degradation and oxidation of electrode compounds through moisture and air attack under ambient conditions are wellestablished in the literature ${ }^{5,39}$ and in this study these degradative species were modeled as $\mathrm{H}_{2} \mathrm{O}$ and $\mathrm{O}_{2}$. Interestingly, we found that the activation barrier for $\mathrm{O}_{2}$ diffusion in graphite (i.e. the coating far from the electrode surface) at very low concentrations is higher than that for Li diffusion in the same graphite coating, that is, $1.0 \mathrm{eV}$ for $\mathrm{O}_{2}$ diffusion as shown in Fig. S6 (ESI $\dagger$ ). In contrast, water was found to diffuse quite easily in graphite with a barrier of only $0.1 \mathrm{eV}$ for jumps between the hollow sites, agreeing well with a relatively low binding energy of water on graphene sheets. ${ }^{40}$ This indicates that the graphite 
coating could act as a filter allowing Li ions to diffuse relatively easily across the carbon coating down into the electrode surface during discharge while preventing the degradative $\mathrm{O}_{2}$ species from reaching the electrode surface, however, without being able to block all degradative species, such as $\mathrm{H}_{2} \mathrm{O}$, which could explain that degradation occurs even in carbon-coated electrode materials.

For the perpendicular-coating- $\mathrm{LiFeBO}_{3}$ interfaces shown in Fig. 4, it is found that a $\mathrm{Li}$ ion positioned at the upper hollow site not terminated by hydrogen atoms has approximately the same energy as when positioned in the lowest hollow site (just above the electrode as shown in the insets of Fig. 7), both being favored by approximately $1 \mathrm{eV}$ compared to the negative-electrode side. NEB results-shown in Fig. 8-yield an activation barrier of $0.2 \mathrm{eV}$ for Li-ion diffusion between the two hollow sites in the $\mathrm{LiFeBO}_{3}$ electrode.

\section{Conclusions}

Using DFT, stability investigations into different graphene-coating structures and the angular orientation of the graphene coating were carried out, as well as the role of defects in the carbon coating in the lithium transport mechanisms. Based on the calculations, it was found that large structural defects and/or significant misalignments between the carbon coatings and the $\mathrm{FeBO}_{3} / \mathrm{LiFeBO}_{3}$ nanoparticles provide mechanisms for facile Li-ion diffusion during charge and discharge. However, significant formation energies of the defects commonly observed in graphene imply low equilibrium concentrations of these defects. Alternative coating geometries where the coating layers were allowed to chemisorb on the electrode surface were investigated, and it was found that the most stable configuration was that in which the coating layer was anchored perpendicularly to the electrode (001) surface with coating-edge $\mathrm{C}$ atoms bonding to surface $\mathrm{B}$ and $\mathrm{O}$ atoms. This optimal coating configuration showed negligible barriers for $\mathrm{Li}$ diffusion from the coating into the electrode surface layer, i.e. this external interface is not predicted to block the Li ions during charge and discharge. Compared to the diffusion of $\mathrm{Li}$ ions at low concentrations in the graphite coating far away from the electrode surface, the diffusion of the electrode-degrading species, $\mathrm{O}_{2}$, was seen to be very low due to a high activation barrier, whereas another electrode-degrading species, $\mathrm{H}_{2} \mathrm{O}$, was seen to easily diffuse in the graphite due to a low barrier of $0.1 \mathrm{eV}$, indicating that the perpendicularly anchored coating configuration partially protects the electrode from degradation under ambient conditions. Furthermore, the Li-ion diffusion in the graphite coating near the electrode was found to be relatively fast due to a low activation barrier of $0.2 \mathrm{eV}$. These findings tally well with experimental observations which show no indications of limitations in the $\mathrm{Li}$ diffusion induced by the application of carbon coating, and instead the overall electrochemical performance is observed to improve.

\section{Acknowledgements}

The research leading to these results has received funding from the European Union's Seventh Framework Programme
(FP7/2007-2013) under grant agreement no. 608575. JMGL acknowledges support from the Spanish Ministry of Economy and Competitiveness under Projects FIS2012-30996 and FIS201346159-C3-1-P and from the Villum Foundation's Young Investigator Programme (4th round, project: In silico design of efficient materials for next generation batteries. Grant number: 10096).

\section{References}

1 R. Chen, S. Ren, M. Knapp, D. Wang, R. Witter, M. Fichtner and H. Hahn, Adv. Energy Mater., 2015, 5, 1-7.

2 C. Delmas, M. Maccario, L. Croguennec, F. Le Cras and F. Weill, Nat. Mater., 2008, 7, 665-671.

3 A. Yamada, N. Iwane, Y. Harada, S. Nishimura, Y. Koyama and I. Tanaka, Adv. Mater., 2010, 22, 3583-3587.

4 S. H. Bo, K. W. Nam, O. J. Borkiewicz, Y. Y. Hu, X. Q. Yang, P. J. Chupas, K. W. Chapman, L. Wu, L. Zhang, F. Wang, C. P. Grey and P. G. Khalifah, Inorg. Chem., 2014, 53, 6585-6595.

5 S.-H. Bo, F. Wang, Y. Janssen, D. Zeng, K.-W. Nam, W. Xu, L.-S. Du, J. Graetz, X.-Q. Yang, Y. Zhu, J. B. Parise, C. P. Grey and P. G. Khalifah, J. Mater. Chem., 2012, 22, 8799-8809.

6 J. C. Kim, C. J. Moore, B. Kang, G. Hautier, A. Jain and G. Ceder, J. Electrochem. Soc., 2011, 158, A309-A315.

7 Z. R. Chang, H. J. Lv, H. W. Tang, H. J. Li, X. Z. Yuan and H. Wang, Electrochim. Acta, 2009, 54, 4595-4599.

8 Y. Ding, Y. Jiang, F. Xu, J. Yin, H. Ren, Q. Zhuo, Z. Long and P. Zhang, Electrochem. Commun., 2010, 12, 10-13.

9 H. Wang, N. Zhao, C. Shi, C. He, J. Li and E. Liu, J. Phys. Chem. C, 2016, 120, 17165-17174.

10 X. Zhou, F. Wang, Y. Zhu and Z. Liu, J. Mater. Chem., 2011, 21, 3353.

11 Y. Janssen, D. S. Middlemiss, S. H. Bo, C. P. Grey and P. G. Khalifah, J. Am. Chem. Soc., 2012, 134, 12516-12527.

12 S. Loftager, J. M. García-Lastra and T. Vegge, J. Phys. Chem. $C, 2016,120,18355-18364$.

13 K. Momma and F. Izumi, J. Appl. Crystallogr., 2011, 44, 1272-1276.

14 S. R. Bahn and K. W. Jacobsen, Comput. Sci. Eng., 2002, 4, 56-66.

15 G. Kresse and J. Furthmöller, Comput. Mater. Sci., 1996, 6, 15-50.

16 P. E. Blöchl, Phys. Rev. B: Condens. Matter Mater. Phys., 1994, 50, 17953-17979.

17 J. P. Perdew, K. Burke and M. Ernzerhof, Phys. Rev. Lett., 1996, 77, 3865-3868.

18 M. S. Islam and C. A. J. Fisher, Chem. Soc. Rev., 2014, 43, 185-204.

19 Y. S. Meng and M. E. Arroyo-de Dompablo, Energy Environ. Sci., 2009, 2, 589.

20 V. I. Anisimov, F. Aryasetiawan and I. Lichtenstein, J. Phys.: Condens. Matter, 1997, 9, 767-808.

21 D.-H. Seo, Y.-U. Park, S.-W. Kim, I. Park, R. Shakoor and K. Kang, Phys. Rev. B: Condens. Matter Mater. Phys., 2011, 83, 205127. 
22 S. L. Dudarev, S. Y. Savrasov, C. J. Humphreys and a. P. Sutton, Phys. Rev. B: Condens. Matter Mater. Phys., 1998, 57, 1505-1509.

23 I. Scivetti and G. Teobaldi, J. Phys. Chem. C, 2015, 119, 21358-21368.

24 J. Wellendorff, K. T. Lundgaard, A. Møgelhøj, V. Petzold, D. D. Landis, J. K. Nørskov, T. Bligaard and K. W. Jacobsen, Phys. Rev. B: Condens. Matter Mater. Phys., 2012, 85, 32-34.

25 E. Bitzek, P. Koskinen, F. Gähler, M. Moseler and P. Gumbsch, Phys. Rev. Lett., 2006, 97, 1-4.

26 H. Jónsson, G. Mills and K. W. Jacobsen, Class. Quantum Dyn. Condens. Phase Simulations - Proc. Int. Sch. Phys., 1998, 385-404.

27 M. Andersen, L. Hornekær and B. Hammer, Phys. Rev. B: Condens. Matter Mater. Phys., 2012, 85405, 1-6.

28 F. Lin, I. M. Markus, D. Nordlund, T. Weng, M. D. Asta, H. L. Xin and M. M. Doeff, Nat. Commun., 2014, 5, 1-9.

29 Y. Shin and K. A. Persson, ACS Appl. Mater. Interfaces, 2016, 8, 25595-25602.

30 F. Yao, F. Güneş, H. Q. Ta, S. M. Lee, S. J. Chae, K. Y. Sheem, C. S. Cojocaru, S. S. Xie and Y. H. Lee, J. Am. Chem. Soc., 2012, 134, 8646-8654.

31 S. Thinius, M. M. Islam, P. Heitjans and T. Bredow, J. Phys. Chem. C, 2014, 118, 2273-2280.
32 R. G. Amorim, A. Fazzio, A. Antonelli, F. D. Novaes and A. J. R. Da Silva, Nano Lett., 2007, 7, 2459-2462.

33 J. Kotakoski, A. V. Krasheninnikov and K. Nordlund, Phys. Rev. B: Condens. Matter Mater. Phys., 2006, 74, 245420.

34 F. Banhart, J. Kotakoski and A. V. Krasheninnikov, ACS Nano, 2011, 5, 26-41.

35 B. Zhang, L. Ming, J. C. Zheng, J. F. Zhang, C. Shen, Y. D. Han, J. L. Wang and S. E. Qin, J. Power Sources, 2014, 261, 249-254.

36 L. Tao, J. R. Neilson, B. C. Melot, T. M. McQueen, C. Masquelier and G. Rousse, Inorg. Chem., 2013, 52, 11966-11974.

37 L. Nilsson, M. Andersen, B. Hammer, I. Stensgaard and L. Hornekær, J. Phys. Chem. Lett., 2013, 4, 3770-3774.

38 K. Persson, V. a. Sethuraman, L. J. Hardwick, Y. Hinuma, Y. S. Meng, A. Van Der Ven, V. Srinivasan, R. Kostecki and G. Ceder, J. Phys. Chem. Lett., 2010, 1, 1176-1180.

39 S. Hamelet, P. Gibot, M. Casas-Cabanas, D. Bonnin, C. P. Grey, J. Cabana, J.-B. Leriche, J. Rodriguez-Carvajal, M. Courty, S. Levasseur, P. Carlach, M. Van Thournout, J.-M. Tarascon and C. Masquelier, J. Mater. Chem., 2009, 19, 3979.

40 J. Ma, A. Michaelides, D. Alfè, L. Schimka, G. Kresse and E. Wang, Phys. Rev. B: Condens. Matter Mater. Phys., 2011, 84, $1-4$. 